\title{
Study on Shuttle Scheduling Problem in Automated Container Terminal
}

\author{
Yifan Shen, Ning Zhao, Weijian Mi, Chao Mi ${ }^{*}$, Xiaohua Zhang and Mengjue Xia \\ Logistics Engineering College, Shanghai Maritime University, Shanghai 201306, China
}

\begin{abstract}
Owing to the shuttle in Mixed Operation Mode of Shuttle-based automated container terminal handles loading, unloading, congregation and delivery operations .simultaneously, conventional scheduling method cannot be applied. Accordingly, this paper proposed a mathematical scheduling model. The objective is to minimize shuttle operation time and deal with loading and unloading with priority while avoiding unbearable waiting time for congregation and delivery tasks. Due to the complexity of this model, a Genetic Algorithm (GA) is designed for effective solutions. Numerical experiment is conducted to examine the key factors of the problem and the performance of the proposed GA.
\end{abstract}

Keywords: Automated container terminal, mixed operation mode, shuttle scheduling, genetic algorithm.

\section{INTRODUCTION}

With the boom of Automated Container Terminal construction, the research of layout design and operations management of Automated Container Terminal has gained more attention. Many handling schemes alone with layout designs have arisen in recent years, among which a shuttle-based handling scheme stands out for better feasibility. This handling scheme matches a shuttle for each yard. This shuttle has much better maneuverability and much lower energy cost than yard crane, and shares vertical transportation workload of yard crane in a faster and more environmentally friendly way. Shuttle operates in Side Operation Mode (SOM), and crane works in End Operation Mode (EOM). This integration of side and end operation is expected to reduce travel distance of yard cranes and increase handling efficiency of the yard. As a new handling scheme, this shuttle-based scheme requires a matched scheduling mode to avoid inefficiency caused by disordered operation dispatch. Accordingly, a new scheduling method is raised. This method has an objective of minimizing shuttle operation time and dealing with loading and unloading with priority while avoiding unbearable waiting time for congregation and delivery tasks, which reasonably expects a higher operation efficiency of the yard.

\section{PROBLEM STATEMENT}

Conventional handling scheme of container terminal uses Quay Crane (QC), truck and Yard Crane (YC) to finish yard operation. The truck picks up a container from QC, move to the loading side of the yard and YC picks up the container and stack it into the yard. As is shown in Fig. (1), the truck operates in Side Operation Mode (SOM). In this mode, YC stacks containers into several adjacent bays consecutively.
Less long range movement saves energy and time, improves efficiency.

Correspondingly, most Automated Container Terminal has a different handling scheme. This scheme uses QC, AGVs and dual YCs as operation facility [1]. According to the change of horizontal transportation facility, external trucks and AGV should have independent operation area to prevent chaos. Vertical block layout has been arose to avoid interference, and dual YCs have been applied to ensure efficiency. As shown in the Fig. (2), when unloading, an AGV picks up a container from QC, move to the shore end of the block to wait for YC to pick up the container and stack it into the yard. Relatively, external trucks load and unload at the land end of the block. YC works in End Operation Mode (EOM) in this handling scheme, for each block, one of the two YC operates at the shore end and the other at the land end.

Compared to SOM, EOM increases YC movement and reduced operation efficiency while isolating operation area of AGVs and external trucks. In order to assure efficiency while avoiding interference, a shuttle-based scheme is raised shown as Fig. (3). Shuttle is a trolley that can carry one container at a time, it has higher speed and lower energy cost than YC. The rail of the shuttle is alongside the block. AGVs are replaced by ALVs that can lift and stack containers. Shuttle operates in a SOM to reduce YC movement, and YCs operates independently in an EOM. This mixed operation mode is called Mixed Operation Mode (MOM).

In MOM, horizontal transportation is separated into two parts, ALVs take care of loading and unloading handling of shore side, and shuttles are in charge of operations in blocks. According to the distinction, scheduling of shuttle should be a new problem.

In the past, a lot of research has focused on improving the scheduling of vehicles in Container Terminal. Bish et al. [2] propose an implementable heuristic algorithms to solve this problem. In Grunow et al. [3], A mixed integer linear programming (MILP) model is formulated to solve a multi- 


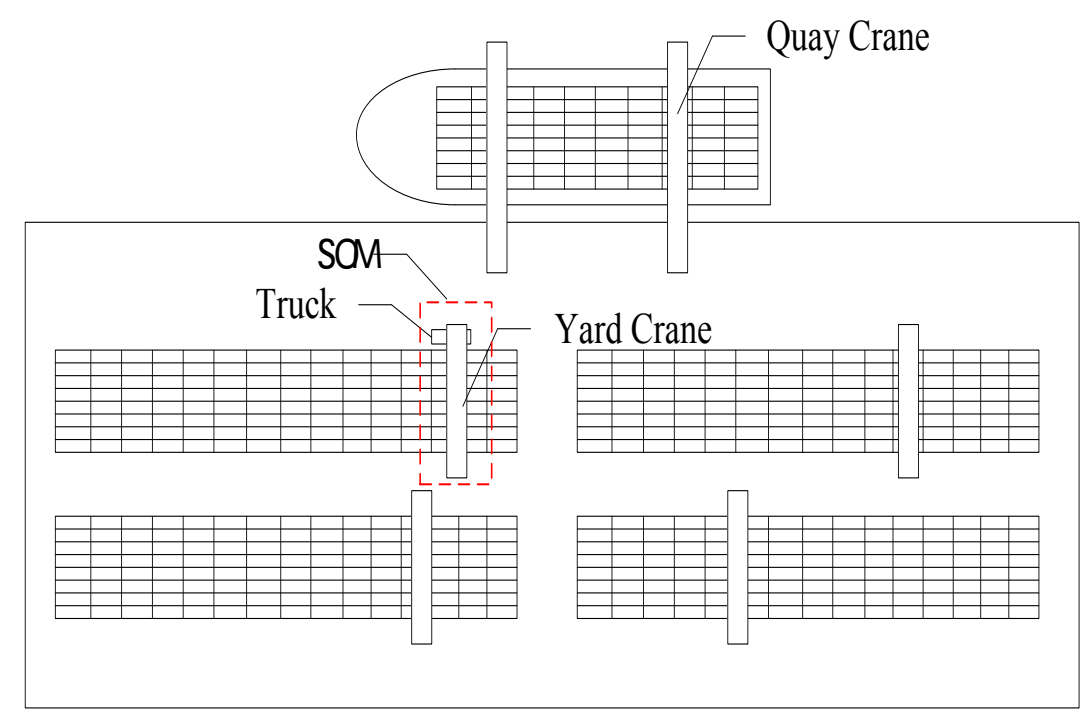

Fig. (1). Layout of traditional container terminal.

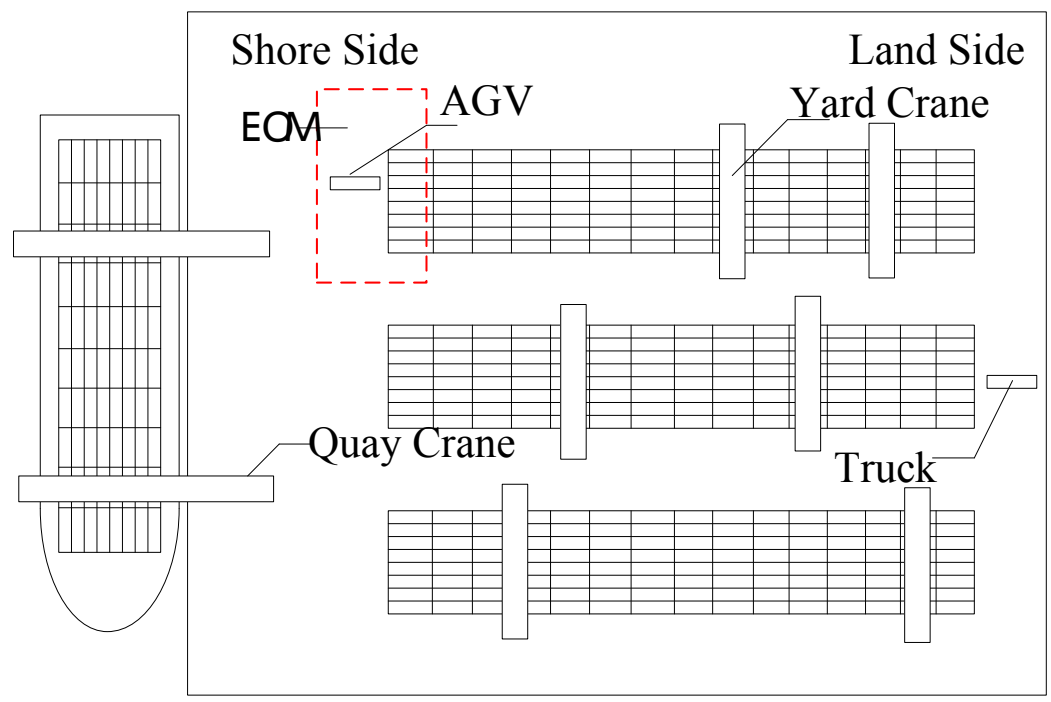

Fig. (2). Layout of automated container terminal.

load AGV dispatching problem. In Lee [4], a hybrid insertion algorithm is designed to solve the truck dispatching problem with the storage allocation problem. Lee et al. [5] consider the problem with the quay cranes and yard cranes capacity. Rashidi et al. [6], solve the problem by a novel algorithm, NSA+, which extended the standard Network Simplex Algorithm (NSA). Moussi et al. [7], develop a heuristic for the dispatching problem of automated lifting vehicles (ALV) which can lift containers from the ground by themselves. Chen et al. [8] study the interactions between crane handling and truck transportation simultaneously, and a three-stage algorithm is developed to solve this problem.

These solutions only takes loading and unloading into consideration, while shuttle handles loading, unloading, congregation and delivery simultaneously. Meanwhile, loading and unloading has a higher priority than congregation and delivery, whereas exceeded handling time of congregation and delivery should be avoided. Thus the objective of scheduling should take priority and waiting time of the four handling operation into consideration while minimizing total handling time. For all these reasons, a model of this scheduling problem is raised accordingly and GA is chosen to solve this model, numerical experiment showed the validity of this model and solution.

\section{MATHEMATICAL FORMULATION}

\subsection{Assumptions}

1. The yard and equipment deal with loading, unloading, congregation, delivery tasks simultaneously, and task type and target bay of each container are given in advance.

2. Container size is ignored. Each container is deemed to be the same size.

3. Relocation and reshuffle are ignored. Each container is lifted from the shuttle and placed onto target slot directly by YC. 


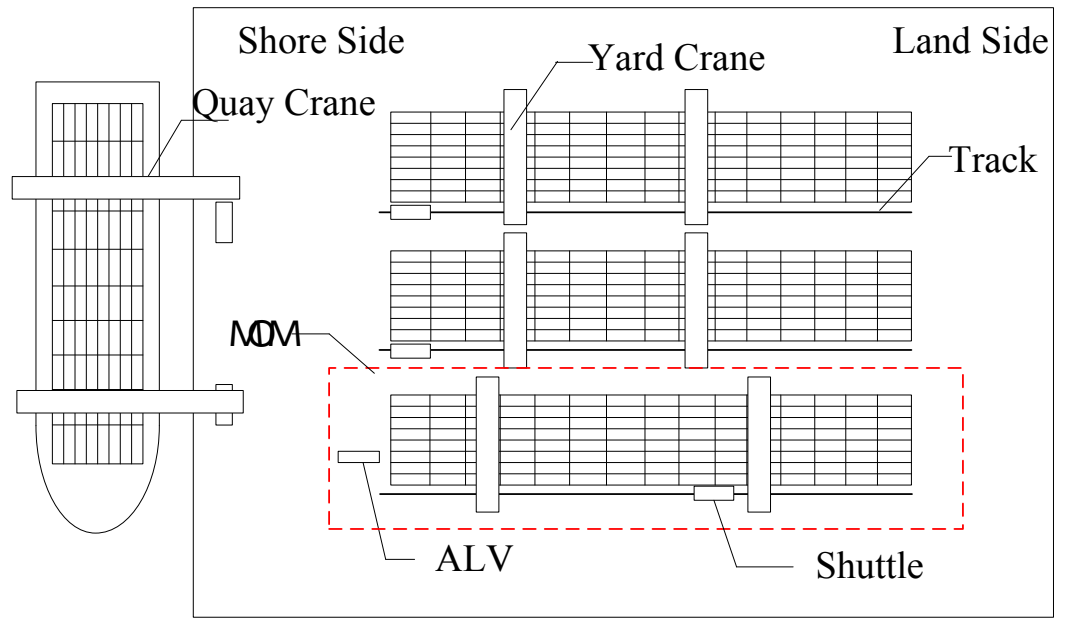

Fig. (3). Layout of automated container terminal based on shuttle handling scheme.

4. Handling time of $\mathrm{YC}$ is ignored.

\subsection{Symbol Definition}

\subsubsection{Dimensions}

$i, j$ : The task $i$ or $j$;

$n$ : The amount of tasks;

$f$ : The head of yard that is the shore side;

$l:$ The tail of yard that is the land side;

$s:$ The shuttle;

$y$ : The yard crane (YC);

\subsubsection{Parameters}

$d_{i f}^{s}$ : The distance between the stopping bay of the shuttle when it finished task ${ }^{i}$ and the shore side $f$;

$d_{f j}^{s}$ : The distance between the operation bays of the shuttle of task $j$ to shore side $f$;

$d_{i j}^{s}$ : The distance between the operation bay of the shuttle of task ${ }^{j}$ and the stopping bay when it finished task ${ }^{i}$;

$d_{l j}^{s}$ : The distance between the operation bay of the shuttle of task ${ }^{j}$ and the land side ${ }^{l}$;

$d_{j f}^{s}$ : The distance between the operation bay of the shuttle of task $j$ and the shore side ${ }^{f}$;

$d_{i l}^{s}$ : The distance between the stopping bay of the shuttle when it finished task ${ }^{i}$ and the land side ${ }^{l}$; $d_{i j}^{s z}$ : Meters the shuttle traveled between the end of task ${ }^{i}$ and the end of $\operatorname{task}^{j}$;

$d_{i j}^{y}$ : Meters the yard crane traveled between the end of task $^{i}$ and the end of task ${ }^{j}$;

$V_{y}:$ The velocity of yard cranes;

$V_{s}$ : The velocity of the shuttle.

$a_{j}: 0-1$ variable, if current task is an unloading task, equals 1 , else equals 0 ;

$b_{j}: 0-1$ variable, if current task is a loading task, equals 1 , else equals 0 ;

$c_{j}: 0-1$ variable, if current task is a congregation task, equals 1 , else equals 0 ;

$e_{j}: 0-1$ variable, if current task is a delivery task, equals 1 , else equals 0 ;

As is shown in Fig. (4), the calculation of travel distance of the shuttle of task $j$ varies between different task types. Take unloading for an example, the total travel distance of unloading task contains two parts, denoted by $d_{i f}^{s}$ hand $d_{f j}^{s}$. Formula $1, d_{i f}^{s}$ is the distance between the stopping bay of the shuttle of last task $i$ and the shore side $f$, and $d_{f j}^{s}$ is the distance between the shore side $f$ and the target bay of task $j$. Similarly, Formula 2 denotes the total travel distance of the shuttle of loading tasks, Formula $\mathbf{3}$ for congregation tasks, Formula 4 for delivery tasks. Formula 5 gives a mixed expression.

Accordingly, the travel distance of the shuttle of any task can be calculated by the following expression: 


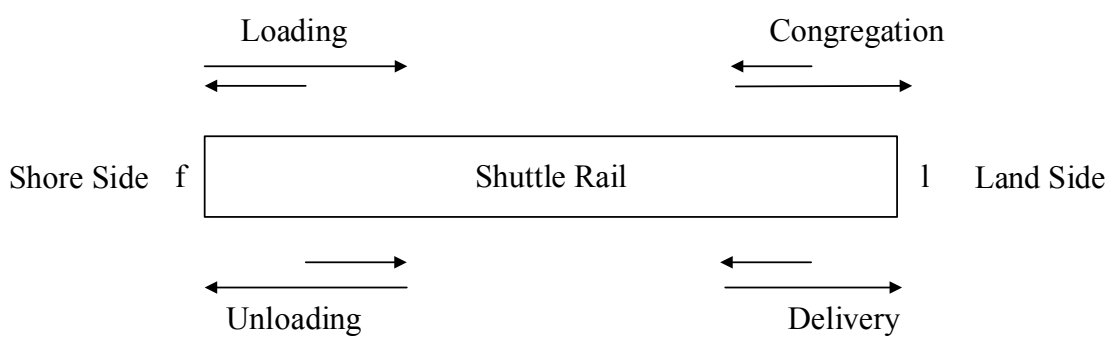

Fig. (4). Process of shuttle execute each task.

$d_{i j}^{s z}=\left(d_{i f}^{s}+d_{f j}^{s}\right) \cdot a_{j}$

$d_{i j}^{s z}=\left(d_{i j}^{s}+d_{j f}^{s}\right) \cdot b_{j}$

$d_{i j}^{s z}=\left(d_{i l}^{s}+d_{l j}^{s}\right) \cdot c_{j}$

$d_{i j}^{s z}=\left(d_{i j}^{s}+d_{j l}^{s}\right) \cdot e_{j}$

$d_{i j}^{s z}=\left(d_{i f}^{s}+d_{f j}^{s}\right) \cdot a_{j}+\left(d_{i j}^{s}+d_{j f}^{s}\right) \cdot b_{j}$ $+\left(d_{i l}^{s}+d_{l j}^{s}\right) \cdot c_{j}+\left(d_{i j}^{s}+d_{j l}^{s}\right) \cdot e_{j}$

\subsubsection{Decision Variable}

$x_{i j}: 0-1$ variable, if the following task of task $i$ is $j$, equals 1 , else equals 0 .

\subsection{Objective Function}

The objective of schedule of shuttle is to obtain an optimized total time consumption of finishing all tasks, and to ensure loading and unloading tasks in a certain extent will take precedence over congregation and delivery tasks. Accordingly, two objective functions have been taken to describe this combined objective.

\subsubsection{Objective 1: Optimized total Time Consumption}

$$
\min t=\sum_{j=1}^{n} \max \left(\sum_{\substack{i=1 \\ i \neq j}}^{n} \frac{d_{i j}^{s z}}{V_{s}} \cdot x_{i j}, \sum_{\substack{i=1 \\ i \neq j}}^{n} \frac{d_{i j}^{y}}{V_{y}} \cdot x_{i j}\right.
$$

Formula 6 denotes the minimization of total time cost. Because the trolley of yard crane starts operation only when the yard crane and the shuttle are both at the target bay, so the time consumption of certain operation cycle should be the longer time of yard crane operation time and shuttle operation time.

\subsubsection{Objective 2: Minimized Punishment for Waiting for Congregation and Delivery}

$$
\min w=\sum_{j=1}^{n}\left[\frac{\sum_{i=1}^{j-1} t_{i j} x_{i j}\left(\mathrm{c}_{j}+e_{j}\right)}{10}\right\rceil
$$

The scheduling should give consideration to both optimizing total time cost and dealing with loading and unloading with priority while avoiding unbearable waiting time for congregation and delivery tasks. This objective is to punish exceeded waiting time for congregation and delivery tasks, Formula 7 calculates the punishment level, this punishment level increases 1 at every 10s, the longer the task waits, the higher punishment level it will get.

Formula 8 is the integrated objective function.

$$
\begin{aligned}
& \min \mathrm{T}=\sum_{j=1}^{n} \max \left(\sum_{\substack{i=1 \\
i \neq j}}^{n} \frac{d_{i j}^{s z}}{V_{s}} \cdot x_{i j}, \sum_{\substack{i=1 \\
i \neq j}}^{n} \frac{d_{i j}^{y}}{V_{y}} \cdot x_{i j}\right) \\
& +\sum_{j=1}^{n}\left[\frac{\sum_{i=1}^{j-1} t_{i j} x_{i j}\left(\mathrm{c}_{j}+e_{j}\right)}{10}\right]
\end{aligned}
$$

\subsection{Constraints}

Four constraints are raised in this model:

$$
\begin{aligned}
& a_{j}+b_{j}+c_{j}+e_{j}=1 \\
& \sum_{i=1}^{n} x_{i j}=1 \\
& \sum_{j=1}^{n} x_{i j}=1 \\
& \sum_{i, j=1}^{n} x_{i j}=n-1
\end{aligned}
$$

Formula 9 ensures only one type of task will the shuttle be doing at a time; Formula $\mathbf{1 0}$ and Formula $\mathbf{1 1}$ guarantee no repeat in task execution; Formula $\mathbf{1 2}$ makes sure every task has been executed at the end of the whole process.

\section{GENETIC ALGORITHEM}

\subsection{Coding}

The solution to the shuttle scheduling problem is to find the best operation sequence of containers. Accordingly, the operation sequence is selected the chromosome code, as is shown in Fig. (5), a chromosome is a job sequence, the task 


\begin{tabular}{rl|l|l|l|l|l|l|l|l|l|} 
Gene Location & 1 & 2 & 3 & 4 & 5 & 6 & 7 & 8 & 9 & 10 \\
Task ID & 5 & 4 & 3 & 10 & 8 & 7 & 2 & 9 & 1 & 6 \\
\hline
\end{tabular}

Fig. (5). Coding.

\begin{tabular}{|c|c|c|c|c|c|c|c|c|c|}
\hline Parent 1 & 2 & 3 & 9 & 5 & 1 & 4 & 6 & 8 & 7 \\
\hline Parent2 & 4 & 5 & 2 & 8 & 3 & 1 & 9 & 7 & 6 \\
\hline
\end{tabular}

Child1 4 \begin{tabular}{l|l|l|l|l|l|l|l|l|}
\hline 4 & 2 & 9 & 5 & 8 & 3 & 1 & 7 & 6 \\
\hline Child2 2 & 5 & 3 & 1 & 4 & 6 & 9 & 8 & 7 \\
\hline
\end{tabular}

Fig. (6). Crossover.

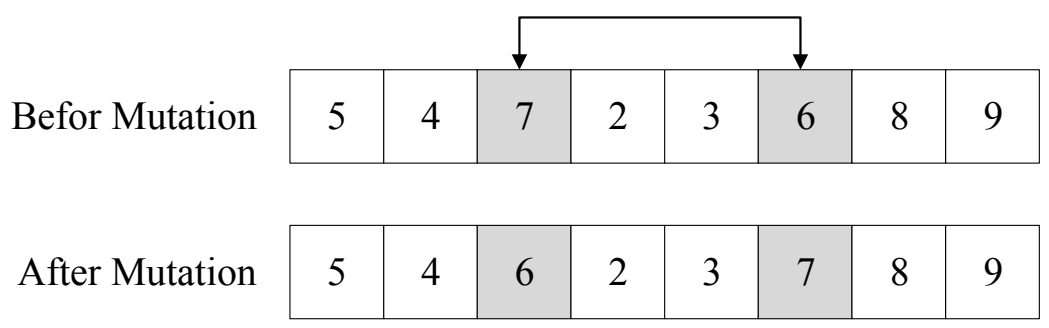

Fig. (7). Mutation.

ID is defined a natural number, thus an chromosome which is a sequence of natural numbers represents an operation sequence.

\subsection{Fitness Function}

GA deserves a non-negative fitness function, the objective function can be selected the fitness function when the objective function is a maximum and non-negative function. Because the objective function of this model is a maximum function and it is non-negative, so that the objective function is selected the fitness function.

\subsection{Selection}

Existing selection operators are Roulette Wheel Selection operator (RWS), Sequential selection operator, Tournament Selection operator (TS), Dominant Selection operator (DSO), Hybrid selection operator, Kin selection operator [9]. RWS is chosen to select the survivals for this problem. The survival probability of certain individual is proportional to its fitness value. The expression of fitness is shown in Formula 13, $n$ is the population size, $i$ and $j$ are individuals.

$$
P_{i}=F_{i} / \sum_{j=1}^{n} F_{j}
$$

\subsection{Crossover}

The procedure for the one point crossover operator used in the current study is summarized here. According to the non-repeatable task execution constraint, every task should be executed once and only once, so any number of tasks are chosen to hold their position, and other task sequence of two parent chromosome is exchanged and fill back to the other parent chromosome according to original sequence as shown in Fig. (6).

\subsection{Mutation}

Inversion mutation, insertion mutation and exchange mutation are common mutation operators to maintain nonrepeat task execution sequence. The procedure for the exchange mutation used in the current study is illustrated in Fig. (7). 


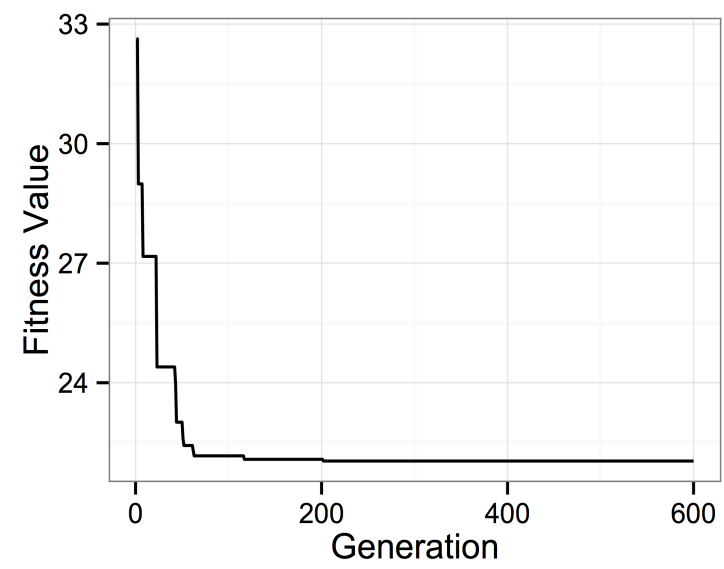

Fig. (8). The convergence curve of specified GA.

\section{NUERICAL EXPERIMENT}

Table 1 shows the input parameters and basic settings of GA, and shuttle tasks are listed in Table 2.

Table 1. Input and GA parameters.

\begin{tabular}{|c|c|c|c|}
\hline \multicolumn{2}{|c|}{ Input parameters } & \multicolumn{2}{c|}{ GA parameters } \\
\hline \hline Key & Value & Key & Value \\
\hline Bay number & 60 & Max generation & 600 \\
\hline Length of each bay & $6.5 \mathrm{~m}$ & Crossover probability & 0.8 \\
\hline Shuttle speed & $250 \mathrm{~m} / \mathrm{min}$ & Mutation probability & 0.05 \\
\hline YC speed & $100 \mathrm{~m} / \mathrm{min}$ & Population & 30 \\
\hline
\end{tabular}

Table 2. Shuttle task list.

\begin{tabular}{|c|c|c|c|}
\hline Target bay & Task type & Target bay & Task type \\
\hline \hline 1 & loading & 57 & unloading \\
\hline 3 & loading & 57 & unloading \\
\hline 3 & loading & 55 & unloading \\
\hline 5 & loading & 59 & unloading \\
\hline 11 & congregation & 55 & unloading \\
\hline 13 & congregation & 43 & delivery \\
\hline 55 & unloading & 31 & delivery \\
\hline 55 & unloading & & \\
\hline
\end{tabular}

\section{RESULT AND ANALYSIS}

This experiment was run on Intel(R) Core(TM) i74870HQ 2.5 GHz PC with 4 GB RAM.
Through this specified GA, the best task sequence of shuttle got is shown as follow:

$$
>15->4->->12->13->15->11->7->2->9->10->3->6->8->14-
$$

As can be seen in the Fig. (8), optimized solution is found after 200 generations within 3 seconds. The optimized fitness value is 20.03 . The optimized fitness value and convergence rate are acceptable.

\section{CONCLUSION}

This paper made a conclusion of handling technologies of current container terminal, both traditional and automated one and further analyzed operation modes among different handling technologies. Accordingly, the paper points out that the distinction of MOM requires a new scheduling plan of horizontal transportation applying to shuttle scheduling. Therefore, a mathematical model of shuttle scheduling is proposed and corresponding genetic algorithm is designed. The significance is as follow:

In light of the particularity of shuttle handling scheme, a new scheduling model is put forward to minimize time of shuttle operation, meanwhile meeting the need of loading and unloading operation without exceeded delaying congregation and delivery operation.

In light of the complexity of solving this scheduling model, this paper designs a genetic algorithm to solve such model and testify the efficiency of the algorithm and feasibility of its result through numerical experiments.

The proposed model and algorithm can serve as a reference to issues and researches on mechanical equipment scheduling concerning MOM.

\section{CONFLICT OF INTEREST}

The author confirms that this article content has no conflict of interest. 


\section{ACKNOWLEDGEMENTS}

This research was supported by "Local University Capacity Promotion Special Program" of the Science and Technology Commission of Shanghai Municipality (No. 13510501800), "Scientific Research Innovation Project" of Shanghai Municipal Education Commission (No. 14ZZ140), the "Ph.D. Innovation Program" of Shanghai Maritime University (No. 2014ycx040), and the Yang Fan Plan for Shanghai Youth Science and Technology Talent (No. 15YF1404900), "Graduate Innovation Fund" of Shanghai Maritime University (No. YXR2014034).

\section{REFERENCES}

[1] J.C. Zheng, H.B. Li, W.N. Xie, Z.J. Li, and R. Yang, "Study on comparison of handling scheme of automated container terminals", Research on Waterborne Transportation, vol. 2, pp. 26-33, 2011.

[2] E.K. Bish, F.Y. Chen, Y.T. Leong, B.L. Nelson, J.W.C. Ng, and D. Simchi-Levi, "Dispatching vehicles in a mega container terminal", OR Spectrum, vol. 27, no. 4, pp. 491-506, 2005. doi: 10.1007/s00291-004-0194-2. load AGVs in highly automated seaport container terminals", $O R$ Spectrum, vol. 26, no. 2, pp. 211-235, 2004.

[4] D.H. Lee, J.X. Cao, Q.X. Shi, and J.H. Chen, "A heuristic algorithm for yard truck scheduling and storage allocation problems", Transportation Research Part E-Logistics And Transportation Review, vol. 45, no. 5, pp. 810-820, 2009.

[5] L.H. Lee, E.P. Chew, K.C. Tan, and Y.A. Wang, "Vehicle dispatching algorithms for container transshipment hubs", OR Spectrum, vol. 32, no. 3, pp. 663-685, 2001.

[6] H. Rashidi, and E.P.K. Tsang, "A complete and an incomplete algorithm for automated guided vehicle scheduling in container terminals", Computers \& Mathematics with Applications, vol. 61, no. 3, pp. 630-641, 2011.

[7] R. Moussi, A. Yassine, A. Kansou, and T. Galinho, "Scheduling of lifting vehicles with time windows in an automated port", vol. 3 , no. 2, 2011

[8] Container terminal, Paper presented at the 2011 4th International Conference on Logistics, LOGISTIQUA'2011, Hammamet, Tunisia.

[9] L. Chen, A. Langevin, and Z.Q. Lu, "Integrated scheduling of crane handling and truck transportation in a maritime container terminal", European Journal of Operational Research, vol. 225, no. 1, pp. 142-152, 2013.

M. Kaya, "The effects of a new selection operator on the performance of a genetic algorithm", Applied Mathematics and Computation, vol. 217, no. 19, pp. 7669-7678, 2011

(C) Shen et al.; Licensee Bentham Open.

This is an open access article licensed under the terms of the Creative Commons Attribution Non-Commercial License (http://creativecommons.org/licenses/by-nc/3.0/) which permits unrestricted, non-commercial use, distribution and reproduction in any medium, provided the work is properly cited. 